\title{
SITUACIÓN DE LOS BUSINESS ANGELS Y DE LAS REDES DE BUSINESS ANGELS EN ESPAÑA ${ }^{1}$
}

State of Business Angels and Networks of Business in Spain

\section{María Concepción Verona Martel Montserrat García Cardona J osé J uan Déniz Mayor}

Resumen: La figura de los Business Angels y de las redes de Business Angels en España es de reciente implantación si se compara con otros países europeos como Reino Unido o Francia. No obstante, esta figura va tomando cada vez una mayor importancia en territorio español.

Palabras claves: Business Angels, redes de business Angels, empresa, JEL: G320.

Este artículo es producto de las líneas del Grupo de Investigación Información Contable, Economía Financiera y Desarrollo Sostenible (INFISOC) de la Universidad de Las Palmas de Gran Canaria-España. 
Abstract: The business angels and business angel networks in Spain are of recent implementation compared to other European countries as UK or France. However, business angels and business angel networks are becoming increasingly important in Spanish territory.

Keywords: Business Angels, Business Angels Networks, Firm, JEL: G320.

\section{Introducción}

Los Business Angels son una figura con larga tradición en los países anglosajones como Reino Unido o Estados Unidos, sin omitir el hecho de que en los últimos años está ganando mucho protagonismo en países como Francia y Alemania. En España todavía son un concepto novedoso, no muy conocido, pero que es de esperar que en los próximos años tenga un mayor protagonismo pues es una importante vía de financiación para las empresas en las primeras etapas de su vida, etapas cruciales de la vida de las mismas. Etapas, por otro lado, en las que las fuentes de financiación tradicionales como es el caso de la financiación bancaria no suele prestar su apoyo financiero a pesar de que son momentos en los que la empresa precisa de más ayuda.

La Asociación Española de Business Angel (AEBAN, 2012a) define al Business Angel como: "un individuo que toma sus propias decisiones de inversión y que aporta su propio dinero, y en ocasiones su tiempo, a empresas no cotizadas promovidas por personas que le son ajenas. Aunque invierte en cualquier etapa del desarrollo, el Business Angel desempeña un papel fundamental en la creación de empresas innovadoras al apoyar a los emprendedores en las fases iniciales del ciclo de vida de sus empresas (semilla y arranque)". 
Situación de los business angels y de las redes de business angels en España

En definitiva, los Business Angels son inversores particulares que invierten su propio dinero, aportando además sus conocimientos, contactos y tiempo en las primeras fases del ciclo de vida de una empresa con el fin de impulsar el desarrollo de la misma.

Asimismo, las redes de Business Angels pueden ser definidas como:

Organizaciones que agrupan a inversores privados (normalmente empresarios, directivos de empresa, ahorradores o emprendedores con éxito) interesados en aportar recursos financieros a nuevos proyectos empresariales de esperado éxito con el objetivo de obtener un beneficio a medio plazo, reencontrar el potencial de los comienzos, disfrutar del espiritu que caracteriza el desarrollo de nuevas empresas y transferir sus conocimientos a los nuevos emprendedores. (Sánchez Solé y Casanueva, 2009: 6-7).

El concepto de red surge de una doble necesidad. Por un lado, la de los Business Angels de encontrar un canal donde poder encontrar y evaluar proyectos de inversión, compartir experiencias y profesionalizar esta actividad. Y, en segundo lugar, la de los emprendedores que buscan en ella a los "mecenas financieros" que permitan que su aventura empresarial bien comience o tenga continuidad y, en el mejor de los casos, éxito. Así, las redes se convierten en el punto de encuentro entre Business Angels y emprendedores, es decir, las redes son las catalizadoras de la oferta y la demanda de capital (Sánchez Solé y Casanueva, 2009: 12). Pero además de ponerlos en contacto, la red también realiza las tareas de:

- Captación y posterior selección de nuevos proyectos susceptibles de recibir financiación;

- Identificación de potenciales Business Angels y de sus expectativas de inversión;

- Mantenimiento del anonimato de las partes hasta el momento del primer contacto; $y$,

- Posibilitar un marco formal para la negociación. 
Los países en los que existe un desconocimiento importante por parte de los empresarios y de los nuevos emprendedores de esta vía de financiación que suponen los Business Angels son numerosos y entre ellos se puede citar a España. De ahí la necesidad de que se realice un esfuerzo para dar a conocer esta figura y las ventajas que tiene para las empresas. Así, por ejemplo, la tarea de promoción de esta figura en España es un objetivo compartido por la Fundación ESBAN (Red Española de Business Angels) y la Dirección General de Política de la PYME.

Arís Coderch et al. (2009: 9) afirman que entorno a la figura de los Business Angels se produce una gran paradoja ya que siendo ampliamente reconocida su función como de vital importancia, entre todas aquellas que promueven el desarrollo económico de una sociedad, es una de las aportaciones más desconocidas y menos estudiadas; su papel es insustituible, por cuanto aporta confianza, en efectivo, en las ideas empresariales de los emprendedores, más allá de cualquier frío análisis y asume riesgos que, por tradición, la propia sociedad y sus entidades financieras no están habituados a asumir. El foco de estos se amplía con una aportación financiera insustituible al mismo tiempo que complementa su actuación con la valiosa combinación de apoyo personal, transmisión de información y experiencia, establecimiento de relaciones e impulso en el ámbito de la gestión empresarial que "nos han permitido acuñar la denominación de las " 3 C" al definir la aportación de capital inteligente del Business Angel: Capital, Conocimientos y Contactos".

Otra cuestión importante es el hecho de que la figura de los Business Angels y de las redes de Business Angels no se encuentran sujetas a ningún tipo de regulación específica en numerosos países, entre ellos está España, lo que además de crear incertidumbre entre los colectivos de potenciales inversores, origina que la financiación aportada por estos no aparezca registrada ni verificada por ningún organismo público o privado. En España se carece de tal regulación pero en otros países de la Unión Europea si la tienen como, por ejemplo, en Francia, país que dispone de una normativa que regula 
esta figura y de un tratamiento fiscal específico. Asimismo, el establecimiento de unas medidas fiscales para este tipo de inversiones también ayudaría a su proliferación, ya que se ha podido comprobar que en aquellos países en que se aplican medidas fiscales para la inversión de los Business Angels, se ha conseguido visualizar y contabilizar su aportación, a la vez que incrementar los fondos dedicados a estas actividades.

El objetivo del presente artículo es poner de manifiesto la situación de los Business Angels y de las redes de Business Angels en España, al mismo tiempo que se hace una breve comparación con el entorno europeo, dado el importante papel que esta figura puede desempeñar en la financiación del tejido empresarial con las consiguientes consecuencias favorables para las empresas y para la economía del país.

El artículo se divide en cuatro secciones. Tras la introducción, se presenta en las secciones dos y tres la situación de los Business Angels y de las redes de Business Angels en España haciendo una breve referencia a la situación de Europa. El artículo finaliza con las conclusiones y la bibliografía.

\section{Situación de los Business Angels en España}

Antes de hacer referencia a la situación en territorio español de los Business Angels se va a proceder a comentar, de forma breve, la situación en Europa, lo que permitirá tener una visión mucho más completa de la situación de este tipo de inversores en España.

De este segmento de la financiación empresarial se tiene información gracias a los estudios y las estadísticas realizados por redes que operan en el conjunto de Europa y a las que los Business Angels se van adhiriendo poco a poco, pues dadas las características de estos inversores entre las que destaca su preferencia por el anonimato, no es nada fácil conseguir información. Estos estudios ponen de manifiesto que la mayor actividad de inversiones realizadas por este 
tipo de inversores en el entorno europeo se concentra en tres países: Reino Unido, Francia y Alemania en los cuales se agrupa el mayor número de redes (Casanovas Ramón, 2011: 110).

$\mathrm{Al}$ analizar a nivel europeo la figura de los Business Angels destacan datos de gran interés como el hecho de que una parte importante de las inversiones realizadas por los Business Angels provienen de Business Angels que no están registrados en ninguna organización o red así como que un tercio de las operaciones fueron efectuadas entre dos o tres Business Angels de forma conjunta (Colomer y Espinet, 2009: 47-48).

A fecha de 2009 se estima que en Europa el número total de inversores activos ronda los 75,000 individuos, con una inversión de capital aproximado de cuatro billones de euros al año. Los Business Angels asociados a redes en Reino Unido se sitúan alrededor de los 5,000 individuos y en Francia alrededor de los 4,000 individuos (EBAN, 2010a).

El informe GEM España 2011 proporciona información de gran interés en relación a los Business Angels en territorio español. Así, se observa cómo en España, desde el inicio de la crisis, la dificultad para obtener financiación se agudiza. Pero un hecho positivo es que los Business Angels siguen estando presentes, incluso han incrementado su participación. En el 2011, el 3.6\% de la población adulta reconoce haber hecho inversiones en empresas de otros lo que supone un incremento del $11.1 \%$ con respecto al año anterior (esta tendencia ascendente se inició en 2008). En una comparativa internacional, teniendo en cuenta estos datos, España tiene un nivel inferior a países como Francia pero superior a países como Alemania, Reino Unido o Dinamarca. A pesar de ello, los datos obtenidos ratifican la importancia que tiene esta fuente de financiación en períodos de crisis, en los cuales las empresas ven recortados los recursos financieros procedentes del sistema crediticio y de los fondos públicos (GEM España, 2011: 65). 
Situación de los business angels y de las redes de business angels en España

Los Business Angels en España son fundamentalmente hombres, mayores de 40 años, con estudios superiores, sin formación específica para emprender, pero cuentan con habilidades y conocimientos necesarios para poner en marcha un negocio, el miedo al fracaso no es un obstáculo para no realizar la inversión y suelen tener una relación de tipo familiar con el emprendedor ${ }^{2}$ (GEM España, 2011: 67).

En relación al volumen medio de inversión por operación cerrada, los datos más precisos que se poseen están referidos al 2008 y la cifra se sitúa alrededor de los 280,000 euros, lo que permite señalar que la media por inversión ha aumentado con respecto a años anteriores; asimismo, y según la evolución que está teniendo el mercado, se espera un crecimiento del volumen en los próximos años (ESBAN, 2008a: 27, 52). En cuanto al números de Business Angels, se considera que está alrededor de 1,500 individuos (p. 25).

En cuanto a la inversión total realizada por los Business Angels, en el año 2009, esta superó la cifra de los 13 millones de euros (Casanovas Ramón, 2001: 110).

Con el fin de que exista un mercado para realizar y cerrar acuerdos entre emprendedores y Business Angels es preciso que haya una oferta (inversores) y una demanda (emprendedores) de capital en el mercado. En España está sucediendo un hecho preocupante y es que la mayoría de los Business Angels con capacidad e iniciativa inversoras no encuentran oportunidades de inversión adecuadas a sus expectativas, $y$, al mismo tiempo, los emprendedores se encuentran con dificultades para identificar inversores potenciales, ya que un importante número de ellos valora en exceso el anonimato y, además, en la mayoría de los casos, no están agrupados en redes (ESBAN, 2008a: 28). De ahí el importante trabajo que han de desempeñar las redes para conseguir solucionar este problema.

2 Los Business Angels, en general, no suelen invertir en proyectos de emprendedores con los que les une una relación de tipo familiar pero en el caso español no es así. El resto de características de los Business Angels españoles son bastante similares a los del resto de Europa. 


\subsection{El TEMA DEL GÉNERO EN EL ÁMBITO DE LOS BuSINESS ANGELS}

La comunidad de Business Angels aún no refleja la diversidad de la sociedad europea. Tanto las mujeres como las minorías étnicas están infrarepresentadas y este hecho debería cambiar con el paso del tiempo.

En Europa menos del 5\% de los miembros de las redes de Business Angels son mujeres y del total de redes europeas el 29\% no cuenta con mujeres entre sus miembros. Pero la situación no es homogénea en toda Europa, pues en Francia y en Polonia al menos un tercio de los Business Angels son mujeres (EBAN, 2010b). En cuanto a España, durante el año 2008, se han identificado 1,465 Business Angels en territorio español de los cuales solo 57 eran mujeres, lo cual se traduce en que tan solo el 3.9\% de la población de Business Angels en España pertenece al género femenino (ESBAN, 2008a: 25).

Un paso importante para conseguir este cambio sería la realización de campañas para animar, de forma particular, a los grupos infrarepresentados de Business Angels potenciales para que entren a formar parte de esta comunidad y sean además activos.

Este hecho también es importante para que los grupos infrarepresentados puedan ser activos en sectores en los que no lo son los Business Angels actuales. Por ejemplo, las mujeres pueden ser más activas en los servicios, en el bienestar y en la educación (las estadísticas actuales confirman bastante estas suposiciones). En Alemania, a pesar de que los Business Angels tanto masculinos como femeninos invertirán mucho (más del 50\%) en tecnologías de la información, las mujeres invertirán más en el sector de servicios que los hombres (Colomer y Espinet, 2009: 80-81).

Existen algunas iniciativas para compensar el desequilibrio en la comunidad de Business Angels. Un ejemplo de estas iniciativas se puede encontrar en Suecia, una red de mujeres profesionales de Hernova (financiada en parte por el Fondo Social Europeo) intenta 
unir a mujeres emprendedoras y a Business Angels y ha iniciado una red de Business Angels que se denomina HerBAN. Esta iniciativa cuenta también con el apoyo de Nutek, la agencia de desarrollo empresarial sueca (Colomer y Espinet, 2009: 80-81).

\subsection{Medidas Para EL FOMENTO DE LOS Business ANGELS EN EsPaÑa}

Con anterioridad se han puesto de manifiesto las ventajas que tienen los Business Angels como fuente de financiación para las empresas. Así que su fomento dentro de un país sería muy beneficioso para el nivel de desarrollo del mismo.

Sánchez Solé y Casanueva (2009: 27-28) señalan que el fomento de esta figura en España como sistema alternativo a la financiación a través del sistema crediticio, del mercado bursátil o del capital riesgo, debería fundamentarse en los siguientes pilares:

a) Creación de un marco normativo definitorio del esquema de funcionamiento de las inversiones de los Business Angels al cual van a dirigirse las políticas de impulso.

b) Identificación de un organismo supervisor del funcionamiento de este nuevo mercado de financiación a fomentar. Dado que se trata de un mercado de financiación a empresas, lo lógico es pensar que dicha función debiera recaer en la Comisión Nacional del Mercado de Valores (CNMV).

c) Establecimiento de una serie de medidas fiscales que fomenten el desarrollo de este mecanismo de financiación. Tales medidas deberían estar enfocadas bajo los siguientes principios:

- Necesidad de acercar la figura del Business Angel a potenciales inversores mediante la creación de estímulos fiscales a la financiación de proyectos empresariales.

- Que el sistema de beneficios fiscales sea de fácil incardinación en el ordenamiento jurídico español. 
- Si el proyecto de inversión es exitoso y las ganancias llegan a la persona física (Business Angel), las ganancias quedarían sujetas a gravamen dentro del esquema impositivo general español.

- Si el proyecto de inversión no es exitoso, debe intentar garantizarse que dicha pérdida económica pueda ser compensada con otras ganancias derivada de otras operaciones como Business Angel o, en último extremo, con otros rendimientos del Business Angel.

- Establecimiento de medidas que impidan la utilización de la figura de forma abusiva o no deseada por el legislador. Estas medias podrían englobar tanto restricciones legales como obligaciones de remisión de información a fin de que la Administración tributaria pueda proceder a su cruce.

En el fondo, el esquema propuesto en este apartado tiene su fundamento, oportunamente adaptado, en la regulación actualmente existente para la figura de financiación más afín al Business Angel ya regulada en España que es la del capital riesgo. Ello es así por tratarse de situaciones totalmente comparables en las que, pese a ubicarse en diferentes momentos de la vida de las empresas, no debieran existir diferencias en lo que respecta a su tratamiento fiscal. Es más, de existir estas, incluso podría defenderse primar al Business Angel frente al capital riesgo al ser mayor el riesgo incorporado a su inversión por realizarse en una etapa más inicial de la vida de la empresa (Sánchez Solé y Casanueva, 2009: 28).

En relación al marco normativo de los Business Angels, este debería definir con suma claridad (Sánchez Solé y Casanueva, 2009: 28):

a) Los agentes o elementos que van a intervenir en este "mercado financiero".

b) Los requisitos que deben reunir dichos agentes. 
Situación de los business angels y de las redes de business angels en España

c) La existencia de un órgano supervisor de este mercado.

d) Las funciones de supervisión y control frente a ciertos agentes que deben, en su caso, existir.

e) Las obligaciones de registro y funcionamiento del órgano supervisor.

f) Las medidas fiscales de fomento de este mercado.

Toda esta regulación podría establecerse mediante un único texto articulado que introdujese también las modificaciones necesarias en la normativa fiscal en vigor actualmente y que pudiera verse afectada.

\section{Situación de las redes de Business Angels en España}

Al igual que el apartado anterior, este se va a desarrollar comentando datos a nivel europeo, lo que permitirá tener una visión más completa de estas redes en territorio español.

El mercado de Business Angels de Reino Unido es el más maduro de Europa. En este país las redes de Business Angels llevan operativas desde hace varios años. Junto a Francia y Alemania es el país que cuenta con el mayor número de redes y con mercados más maduros.

Hasta el 2008 se habían identificado en Europa 297 redes (en 1999 eran 50 redes). En países como Francia, España y Portugal el número de redes ha aumentado considerablemente a lo largo de los últimos tres años. En Europa Central y del Este el sector muestra una tendencia de desarrollo lenta, aunque constante, ejemplo de ello, es el hecho de que durante 2008 se hayan creado dos redes en Bulgaria y una en Ucrania (Colomer y Espinet, 2009: 100-102). En general, el número de redes en Europa en 2008 se ha incrementado y los resultados del 2009 confirman la tendencia ascendente en la creación de redes de Business Angels (EBAN, 2008; EBAN, 2009; EBAN, 2010a). Los datos obtenidos apuntan a que en 2010 en Europa habrá más de 350 redes (EBAN, 2010c). 
Tanto en España como en el resto de Europa las redes prestan una serie de servicios a los Business Angels y a los emprendedores. Pero la comparación entre las redes españolas y las del resto de Europa muestra una tendencia de las segundas a enfocar sus servicios al Business Angel, en cambio, las españolas dirigen sus servicios a los emprendedores. El mercado europeo se encuentra en una fase más desarrollada y por lo tanto cuenta con un sector inversor mucho más maduro, el cual acumula más experiencia y mayores niveles de actividad (ESBAN, 2008a: 51).

En cuanto a la especialización de las redes por sectores se observa que mientras a nivel europeo, a fecha de 2008, el $88 \%$ de las redes no se han especializado en actividades económicas concretas, el porcentaje para España es inferior (73\%), lo que indica un grado de especialización ligeramente superior en el caso español en comparación con el resto de Europa (ESBAN, 2008b: 125). Esto se debe a la fuerte dependencia que tienen las redes españolas con sus entidades constituyentes, organismos que en la mayoría de casos son parques científicos, tecnológicos o universidades, que por su naturaleza ofrecen proyectos especializados en algún sector determinado (p. 137).

En relación al ámbito de actuación de las redes, a nivel europeo la tendencia es bastante similar al caso español, al menos hasta 2008. En este sentido se observa que el $57 \%$ de las redes en el resto de Europa ofrecen una cobertura autonómica o regional $(52 \%$ en España), el 24\% tiene un alcance estatal (25\% en España) y el 2\% indica poseer un ámbito de actuación internacional (en este caso el porcentaje español es mayor, un 8\%) (ESBAN, 2008b: 127). Además, se ha observado que las inversiones locales se han incrementado en detrimento de las nacionales y regionales (EBAN, 2009).

En lo referente a las fuentes de financiación de las redes, con datos de 2008, se observa un comportamiento inverso entre las redes españolas y las restantes redes europeas en cuanto a la obtención de recursos mediante fuentes de financiación de carácter externo, pues las redes españolas utilizan principalmente esta vía de financiación 
Situación de los business angels y de las redes de business angels en España

en cambio no es así en las restantes redes europeas. $\mathrm{Y}$, en cuanto a la financiación interna, se le da una mayor relevancia a esta forma de financiarse entre las restantes redes europeas que entre las españolas. Así a nivel europeo, se observa que la principal fuente de financiación de las redes son las subvenciones a fondo perdido (64\%) mientras que en España son las aportaciones de las entidades constituyentes o socios (51\%). Este dato muestra que las restantes redes europeas pueden optar a un mayor volumen de capital a través de este tipo de financiación en comparación con las redes españolas, quienes disponen de un menor número de programas de ayuda y recursos a fondo perdido para financiar sus actividades. En este sentido, la falta de una correcta regulación y mejora del trato fiscal del sector en España perjudica a las redes nacionales frente a las restantes europeas, que se ven aventajadas en este y otros aspectos. Por otro lado, destaca el hecho de que solo en el 15\% de los casos de las restantes redes europeas se financian a través de aportaciones de sus entidades constituyentes o socias, hecho que refleja una menor dependencia de los recursos externos (ESBAN, 2008b: 132-133).

En cuanto al patrocinio, se observa que es un recurso financiero mucho más utilizado a nivel europeo, el cual triplica al español, dato que muestra que en el modelo de ingresos europeos incide mucho más la parte interna y directa de la actividad desarrollada por la red en comparación con el caso español. En esta línea, se observa que los resultados obtenidos a nivel europeo en las categorías de cuotas de inversores, de emprendedores y de éxito son muy superiores con respecto a los españoles. La mayor madurez y regulación del sector en el caso europeo, basada en una cultura de apoyo y contribución a las redes, propician una participación más activa de los agentes adheridos a la red, inversores y emprendedores, que reconocen la importancia de la existencia y de las actividades de las redes de Business Angels (ESBAN, 2008b: 132-133).

Haciendo un mayor hincapié en las fuentes de financiación de las redes españolas, con datos de 2008, se puede observar que la principal fuente de financiación de las redes españolas proviene de 
las entidades constituyentes o socias $(51 \%)$ y, en segundo lugar, de subvenciones (39\%). Por otro lado, si se analizan las fuentes de financiación generadas por la actividad directa de las redes, destaca el patrocinio, con un $24 \%$. A nivel íntegramente interno, destacan como principales fuentes de financiación las cuotas de los inversores adheridos a la red (27\%), las cuotas de éxito que aplican a las inversiones realizadas $(21 \%)$ y las cuotas de los emprendedores $(12 \%)$. La diferencia entre cuotas de emprendedores e inversores es consecuencia de la práctica generalizada de fijar cuotas más altas a los inversores, considerados con mayor poder económico, no fijándose en algunos casos, cuotas para los emprendedores (ESBAN, 2008b: 131). En suma, las redes de Business Angels en España se financian principalmente a través de recursos externos, concretamente, a través de entidades constituyentes, subvenciones y patrocinios. Este hecho se debe básicamente a que:

- Las redes en España se encuentran en fases iniciales de desarrollo y, por lo tanto, centran sus actividades en fomentar la concienciación de los inversores y la captación de proyectos; $y$,

- La fuerte dependencia de las redes españolas con sus entidades constituyentes y/o fundadoras (pp. 138-139).

Dentro del marco del Third Multiannual Programme for SMEs (1997-2000) se puso en marcha en 1998 el Programa Piloto de la Red de Business Angels, uno de los frutos de este programa fue la constitución en 1999 de la European Business Angels Network (EBAN) con sede en Bruselas. La EBAN es una asociación sin ánimo de lucro que actúa como red de redes a nivel europeo con el objetivo de liderar y representar este sector, al mismo tiempo que lucha por incrementar la visibilidad del valor añadido que suponen los Business Angels así como las redes de Business Angels en el denominado mercado de la inversión privada informal; también, ha influido en las políticas públicas para que apoyen las actividades desarrolladas por las redes de Business Angels. 
Situación de los business angels y de las redes de business angels en España

Actualmente, la EBAN incluye actualmente a más de 100 redes de Business Angels de más de 25 países y más de 20,000 Business Angels. Las redes de Business Angels asociadas a la EBAN son de dos tipos: nacionales y regionales, siendo estas últimas la mayoría; destacando el hecho de que no todos los países tienen sus redes en la EBAN, por ejemplo, España es uno de los países que tiene una mayor presencia.

Los principales objetivos de la EBAN se pueden concretar en los siguientes:

a) Fomentar el intercambio de experiencias y la comunicación de mejores prácticas entre las redes de Business Angels.

b) Promover el reconocimiento de las redes de Business Angels y de la función que estos desempeñan en la economía.

c) Contribuir al desarrollo y puesta en práctica de programas locales, regionales y nacionales dirigidos a estimular la creación y consolidación de unas condiciones positivas para la actividad de los Business Angels.

Las redes de Business Angels deben tener un código de conducta, por ejemplo, la EBAN tiene un código de conducta, el cual es seguido también por otro tipo de redes en el continente europeo ${ }^{3}$.

En cuanto a España, el concepto de redes de Business Angels aún es muy novedoso si lo comparamos con el nivel de desarrollo en otros países como el Reino Unido, Francia o Alemania. Así, por ejemplo, en 1999 en Reino Unido, que es el mercado más maduro en Europa de Business Angels, había 49 redes y en España ninguna. En el año 2004 las redes en España ascendían a 11 redes y en el Reino Unido a 101 redes, y el número ha seguido subiendo en ambos países pero la diferencia entre ellos poco a poco va disminuyendo.

3 En la página web de EBAN se encuentra publicado su código de conducta (EBAN, 2012). 
El hecho de que las redes sean las entidades catalizadoras del mercado entre emprendedores y Business Angels, hace que las mismas hayan surgido por el apoyo de los principales agentes comprometidos con la creación de empresas. Así, las redes existentes en territorio español tienen marcados orígenes del mundo académico e investigador, patronal o gubernamental (ya sea local, autonómico o estatal).

Hasta principios del año 2004 en España no existía ninguna red que actuase como entidad agrupadora de las mismas, es decir, como red de redes. Esta situación cambia al crearse en dicho año, bajo la forma jurídica de fundación privada, sin ánimo de lucro y como entidad adherida a la European Business Angels Network (EBAN), la Red Española de Business Angels (ESBAN). La ESBAN agrupa a todas las redes públicas y privadas de ámbito regional o local españolas, así como todas aquellas entidades con fines coincidentes con la fundación que quieran participar en las actividades que en ella se organicen. Y actúa como una red de redes no como una red de Business Angels, no disponiendo de un código de conducta. Sus entidades fundadoras fueron: Business Angels Network Catalunya, Confederación Empresarial Comarcal de Terrassa y Fundación Privada Cecot Innovación.

La ESBAN favorece, por un lado, el fomento y desarrollo de las redes de Business Angels y, por el otro, el contacto entre los inversores y emprendedores, contribuyendo a la creación y consolidación de las pequeñas y medianas empresas. Asimismo, es una de las entidades fundadoras de la World Business Angels Association (WBAA) y cuenta con el apoyo de la Dirección General de Industria y de la Pequeña y Mediana Empresa.

Los objetivos que debe cumplir la red ESBAN se pueden concretar en los siguientes (ESBAN, 2012):

a) Promover y difundir el concepto Business Angel como una alternativa de financiación adaptada a las necesidades de los emprendedores. 
Situación de los business angels y de las redes de business angels en España

b) Desarrollar el gran potencial latente que pueden aportar los Business Angels al conjunto de la economía, así como promover la inversión con el fin de favorecer la creación y el desarrollo de nuevas empresas.

c) Fomentar el desarrollo de redes de Business Angels en España $\mathrm{y}$ defender sus intereses.

d) Colaborar con la Administración para el desarrollo de un marco jurídico y fiscal adaptado a las necesidades de los Business Angels.

En relación a las funciones que debe desempeñar la red ESBAN se pueden citar las siguientes:

a) Representación institucional, a nivel nacional y europeo, del resto de redes regionales y locales con independencia de su ámbito de actuación.

b) Asesoramiento en la creación y puesta en marcha de nuevas redes regionales o locales:

- Desarrollo de la red

- Proceso metodológico

- Plataforma tecnológica 4

- Actividades de promoción y formación

c) Liderazgo de proyectos conjuntos entre las distintas redes

d) Transferencia de planes de negocio entre las distintas redes

e) Edición y actualización de estudios, documentación, noticias $\mathrm{y}$ boletines.

4 Con el objetivo de integrar el modelo operativo de cada red regional o local, desde la Red Española de Business Angels se pone a disposición de las distintas redes una plataforma tecnológica que, aparte de realizar todas las funciones de una página web descriptiva e informativa, permite gestionar la base de datos de inversores y emprendedores con la opción de: a) Inscribirse como socio Business Angel o como emprendedor en cada red; b) Consultar, por parte de los inversores, todos los proyectos adscritos en las diferentes redes; c) Permitir un matching (proceso de toma de contacto entre el Business Angel y el emprendedor) de proyectos on-line; y, d) Enviar un boletín de proyectos a todos los inversores. Estas plataformas interactivas están en funcionamiento actualmente en algunas redes regionales pero no en todas. 
f) Organización de actividades y actos:

- Congresos

- Foros de inversión

- Workshops

- Formación para Business Angels con el fin de capacitarlos para el proceso de inversión en proyectos empresariales

España cuenta con distintas redes distribuidas por distintas comunidades autónomas, siendo tres comunidades las que cuentan con un mayor número de ellas, que son: Cataluña, Comunidad Valenciana y Comunidad de Madrid, por este orden. En total, para el 2008 existían en España 40 redes, cuyo número en 2012 se eleva a 56 redes.

Realmente en España aparece la primera red de Business Angels en el año 2002, aunque otras sociedades de desarrollo económico o Centros Europeos de Empresas Innovadoras fueron fundadas con anterioridad siendo sus actividades en el sector posteriores a esta fecha, de ahí que muchas estadísticas reflejen que ya había redes en territorio español en el año 2000 (ESBAN, 2008b: 121). Esta primera red se creó en Cataluña y es la red BANC (Business Angels Network Catalunya), con ámbito autonómico, de naturaleza privada, sin ánimo de lucro y sin especialización en ningún sector. Esta red fue creada por la Patronal CECOT, la Fundación CP'AC y el Institut Català de Finances Holding, SA (ESBAN, 2008b: 43).

Para el 2004, la presencia de redes empieza a extenderse por todo el territorio español. Así, se localizan las primeras redes con entidad jurídica propia en las comunidades autonómicas de Cataluña, Madrid, Canarias, Extremadura y Galicia, llegando a alcanzar un total de 11 redes.

En el 2006, se experimenta un importante auge de las redes en España, intensificándose en comunidades como Cataluña, Comunidad Valenciana, Madrid y Galicia, y apareciendo en nuevas comunidades 
Situación de los business angels y de las redes de business angels en España

como Cantabria, La Rioja, Andalucía e Islas Baleares. En concreto, a partir del año del 2005 se crearon el 65\% de las redes identificadas en España (ESBAN, 2008b: 122). En el 2008 se observa una extensión casi total de las redes en el territorio español, confirmándose así la creciente actividad del sector y el carácter localista de las inversiones y de las redes, además es de señalar que la mayoría de las redes españolas no se ha especializado en sectores económicos concretos, al contrario incorporan proyectos y realizan inversiones en cualquier sector económico (pp. 123, 125).

Si se analiza el tipo de sectores económicos con un mayor grado de inversión por parte de las redes españolas, se observa una clara concentración en tres sectores: TIC/software/multimedia, biotecnología y energías renovables. Una explicación a este hecho se encuentra en el carácter localista de las redes e inversiones realizadas. Es habitual que las redes españolas se instalen en zonas industriales, viveros de empresas, parques científicos y tecnológicos, universidades o núcleos similares con el fin de aproximarse a las fuentes donde captar los proyectos empresariales, lo que conlleva un mayor flujo de proyectos que provienen de sectores donde se encuentran establecidas y por tanto una especialización en determinados sectores económicos. En este sentido, se observa que un 56\% de las redes españolas que han declarado especializarse en algún sector económico concreto están ubicadas dentro o en las proximidades de los núcleos mencionados, que si bien no es un factor determinante, demuestra la importancia de la localización en el comportamiento de las redes (ESBAN, 2008b: 126-127), y, además, tales redes se concentran en los sectores de las TIC y el biotecnológico (p. 137).

En relación a la forma jurídica de las redes, a fecha de 2008, el 38\% de las redes españolas declaran poseer entidad jurídica propia, de las cuales el $28 \%$ pertenece al sector privado y el $10 \%$ son iniciativas públicas. De ese $38 \%$ de redes que tienen entidad jurídica propia, se observa que un $47 \%$ son sociedades mercantiles, constituidas en su mayoría, como sociedades limitadas, otro $47 \%$ se constituyen como asociaciones y un $6 \%$ son fundaciones. Por otro lado, el resto de las 
redes identificadas $(62 \%)$ no poseen entidad jurídica propia y, por tanto, están gestionadas por otras organizaciones, que aportan su estructura, a nivel de recursos humanos y financieros, para mantener y desarrollar las actividades de la red (ESBAN, 2008b: 128-129).

Estas redes que no poseen entidad jurídica propia, a fecha de 2008, son gestionadas por una gran diversidad de organismos gestores. En términos generales, se observa que la mayoría de estos organismos son Centros Europeos de Empresas e Innovación (CEEI) y entidades públicas, con un $24 \%$ y $20 \%$ respectivamente, les siguen, por orden de prioridad, las sociedades mercantiles (16\%) y las escuelas de negocios $(12 \%)$. En este sentido, adquieren especial relevancia los organismos considerados públicos, como los CEEI, en especial de Baleares y la Comunidad Valenciana, las sociedades para el desarrollo económico y las mismas instituciones públicas, como ayuntamientos, juntas o cámaras de comercio. En conjunto, representan el 52\% de las entidades gestoras de redes, hecho que "ratifica la fuerte apuesta desde la Administración Pública hacia el desarrollo y crecimiento del sector y de la actividad económica en general" (ESBAN, 2008b: 129-130). Por otro lado, destaca el importante papel de las instituciones educativas en la gestión de redes españolas, así un $20 \%$ de las redes son gestionadas por reconocidas y prestigiosas universidades $(8 \%)$ y escuelas de negocios $(12 \%)$, las cuales han integrado las actividades de las redes en su estructura "en otra demostración del éxito y fortaleza de las redes como catalizadoras de proyectos empresariales y excelentes trampolines de innovación” (p. 130).

En cuanto al ánimo de lucro, es importante resaltar, que las redes en España se distribuyen de una forma bastante homogénea pues un $53 \%$ son redes sin ánimo de lucro frente al 47\% que son entidades con ánimo de lucro (ESBAN, 2008b: 129).

En este apartado es necesario hacer referencia a la Asociación Española de Business Angels Networks (AEBAN) creada en 2008 como una asociación sin ánimo de lucro, independiente y organizada democráticamente que asocia a las principales Redes de Business 
Angels que existen en España. Su misión principal es promocionar la actividad de los Business Angels y de las Redes de Business Angels en el territorio español como parte fundamental del sistema de $\mathrm{I}+\mathrm{D}+\mathrm{i}$ nacional. A fecha de 2012, AEBAN asocia un total de 26 Redes de Business Angels con sede principal en 11 Comunidades Autónomas, las cuales, a su vez, asocian cerca de 400 inversores que en el último año movilizaron recursos por valor de 20 millones de euros (AEBAN, 2012b).

Los objetivos perseguidos por la AEBAN se centran en los siguientes (AEBAN, 2012b):

a) Aglutinar a todas las Redes de Business Angels de España.

b) Servir de foro de intercambio de información, experiencias y proyectos entre representantes de Redes de Business Angels, Administraciones Públicas, instituciones de enseñanza y cualquier otra institución o entidades interesadas en los fines de la Asociación.

c) Colaborar en la promoción e intercambio de proyectos de inversión.

d) Ejercer un papel de interlocutor de las Redes de Business Angels con las instituciones públicas o privadas, así como con Administraciones Públicas con el objetivo de promover la actividad de los Business Angels.

e) Relacionarse con otras asociaciones y redes en ámbitos internacionales y, en especial, con EBAN.

f) Identificar, promover y compartir las "mejores prácticas" en el desarrollo de las actividades de las Redes de Business Angels y en los procesos de inversión.

g) Fomentar el aprendizaje y la actualización constante de conocimientos entre los socios de la Asociación. 
h) Promover la reflexión sobre cuestiones específicas que afectan a los Business Angels.

i) Difundir información sobre los Business Angels y las Redes de Business Angels.

j) Promover la realización de estudios periódicos sobre el mercado de la inversión privada.

En España todavía hay un largo camino por recorrer para que los Business Angels se conviertan en una fuente de financiación al alcance de los nuevos empresarios tal y como ocurre en el resto de países europeos (ESBAN, 2008a: 25). Pero si se tiene presente el comportamiento del sector hasta el momento el futuro es bastante alentador con los beneficios que ello conlleva para las empresas españolas y para la economía del país en general.

\section{Conclusiones}

Los Business Angels y las redes de Business Angels son figuras que han surgido en el mercado de fuentes de financiación para las empresas en el entorno español en un período relativamente reciente si se compara con los mercados más maduros del resto del continente europeo. A pesar de ello, su importancia va creciendo con el paso de los años y a medida que se toma conciencia de las importantes ventajas de la existencia de esta figura como fuente de fondos para las empresas, especialmente en las etapas iniciales de la vida de las mismas. A la vista de los datos que se han podido recolectar sobre esta figura, el futuro de los Business Angels en España parece prometedor. A medida que vaya creciendo la información con la generación de nuevas redes se podrá determinar si esta figura está en vías de consolidación en el territorio español. 
Situación de los business angels y de las redes de business angels en España

\section{Referencias bibliográficas}

Asociación Española Business Angels (AEBAN) (2012a). Business Angels. Madrid: Asociación Española Business Angels (AEBAN). Recuperado de http://www.aeban.es

Asociación Española Business Angels (AEBAN) (2012b). ¿Qué es AEBAN? Madrid: Asociación Española Business Angels (AEBAN). Recuperado de http://www.aeban.es

Arís Coderch, G., Clos Nogueras, J., Colomer Espinet, A., Rodríguez Ripollès, J., Munk, C. \& White, M. (2009). Análisis de tributación comparada de la figura de los Business Angels en Europa. Estudio para la propuesta de medidas fiscales de fomento. Madrid: Dirección General de Política de la Pequeña y Mediana Empresa, Ministerio de Industria, Turismo y Comercio, Gobierno de España.

Casanovas Ramón, M. (2011). Alternativas de financiación no tradicionales para PYMES. Revista de Contabilidad y Dirección, 12, 95-112.

Colomer i Espinet, A. (2009) (Dir.). Los Business Angels, innovando en la cultura de financiación de las empresas. Madrid: Dirección General de Política de la Pequeña y Mediana Empresa, Ministerio de Industria, Turismo y Comercio, Gobierno de España.

European Business Angels Network (EBAN) (2008). Statistics compendium 2008. Bruselas: European Business Angels Network.

European Business Angels Network (EBAN) (2009). Statistics compendium 2009. Bruselas: European Business Angels Network.

European Business Angels Network (EBAN) (2010a). Statistics compendium 2010. Bruselas: European Business Angels Network. 
European Business Angels Network (EBAN) (2010b). EBAN White Paper. (November). Bruselas: European Business Angels Network.

European Business Angels Network (EBAN) (2010c). EBAN White Paper. (october). Bruselas: European Business Angels Network.

European Business Angels Network (EBAN) (2012). EBAN Members' Code of Conduct. Bruselas: European Business Angels Network. Recuperado de http://www.eban.org/?page_id=414

Global Entrepreneurship Monitor España (GEM España) (2011). Informe GEM España 2011. Madrid: Global Entrepreneurship Monitor España (GEM España)

Red Española de Business Angels (ESBAN) (2008a). Redes españolas de Business Angels: Investigación de procedimientos y volumen de mercado. Madrid: Dirección General de Política de la Pequeña y Mediana Empresa, Ministerio de Industria, Turismo y Comercio, Gobierno de España.

Red Española de Business Angels (ESBAN) (2008b). Naturaleza de las redes de business angels existentes en España y principales características de los agentes del mercado. Madrid: Dirección General de Política de la Pequeña y Mediana Empresa, Ministerio de Industria, Turismo y Comercio, Gobierno de España.

Red Española de Business Angels ESBAN (2012). ¿Qué es ESBAN? Recuperado (s. f.) de http:/ / esban.cecot.org/que-es-esban/

Sánchez Solé, S. \& Casanueva, F. (2009). Propuesta de medidas fiscales de fomento de la figura de los Business Angels en España. Madrid: Dirección General de Política de la Pequeña y Mediana Empresa, Ministerio de Industria, Gobierno de España. 


\section{María Concepción Verona Martel}

Licenciada en Ciencias Económicas y Empresariales, Universidad de Las Palmas de Gran Canaria, España; PH. D. en Ciencias Económicas y Empresariales. En esta misma institución realiza su PH. D. Es profesora titular de la Facultad de Economía, Empresa y Turismo, de la Universidad de Las Palmas de Gran Canaria, España; miembro del Grupo de Investigación Información Contable, Economía Financiera y Desarrollo Sostenible (INFISOC) de la. Universidad de Las Palmas de Gran Canaria, España.

Email: cverona@defc.ulpgc.es

\section{Montserrat García Cardona}

Graduada en Turismo por la Universidad de Las Palmas de Gran Canaria, España. 


\section{José Juan Déniz Mayor}

Licenciado en Ciencias Económicas y Empresariales, Universidad de Las Palmas de Gran Canaria, España; PH. D. en Ciencias Económicas y Empresariales. En esta misma institución realiza su PH. D. Es profesor titular de la Facultad de Economía, Empresa y Turismo, de la Universidad de Las Palmas de Gran Canaria, España; miembro del grupo de Investigación Información Contable, Economía Financiera y Desarrollo Sostenible (INFISOC), Universidad de Las Palmas de Gran Canaria, España.

Email: jdeniz@defc.ulpgc.es

Recibido: 15/12/2012

Aprobado: 22/02/2013 